\title{
An Optimization Method for the Main Influence Factors on the Performance of Aerostatic Spindle
}

\author{
Dongju Chen (D), You Zhao, Ri Pan (D), and Jinwei Fan \\ Beijing Key Laboratory of Advanced Manufacturing Technology, Beijing University of Technology, Beijing 100124, China \\ Correspondence should be addressed to Ri Pan; panri@bjut.edu.cn
}

Received 11 January 2019; Revised 13 March 2019; Accepted 2 April 2019; Published 15 April 2019

Academic Editor: Chengzhi Shi

Copyright ( 92019 Dongju Chen et al. This is an open access article distributed under the Creative Commons Attribution License, which permits unrestricted use, distribution, and reproduction in any medium, provided the original work is properly cited.

\begin{abstract}
This paper proposes a novel optimization method to improve the performance of aerostatic spindle. The optimization process is based on the main factors affecting the load and stiffness of aerostatic spindle, which are determined by cross-correlation analysis. The maximum values of load capacity and stiffness are obtained from the relationship between the optimization function and the main influence factors. After optimization, the corresponding natural frequency has been improved, which indicates that the optimization method proposed in this paper is more efficient and reliable in the field of precision machining. The results will enforce the industrial application of aerostatic spindle system.
\end{abstract}

\section{Introduction}

The aerostatic spindle is widely used in precision and ultraprecision machining by the advantages of low friction and high accuracy [1]. When the aerostatic bearing is working, the air supply is provided by an external air compressor. Then, the gas enters the air cavity through the orifice to form a layer of gas film which can support the workpiece to machine and achieve frictionless lubrication. The bearing capacity of the aerostatic spindle mainly comes from the gas film between the aerostatic bearing and the spindle. For ultraprecision machining, the stability of aerostatic spindle should be guaranteed. In fact, the performance of the aerostatic spindle is completely different due to some factors, such as structural parameters of air cavity, orifice diameter, gas film thickness, and air supply pressure. And the performance of the aerostatic spindle is a key factor affecting the quality of the machine [2]. Besides, it will be also directly reflected to the surface of the workpiece and affect the quality of the workpiece. Therefore, it is important to study the performance of the aerostatic spindle to improve the precision of ultraprecision machining.

There are two major aspects for the optimization of aerostatic spindle: one focuses on the static characteristics analysis of aerostatic spindle to optimize basic parameters
[3]. Aguirre et al. [4] proposed an active compensation strategy to achieve nanometer position control, which can improve the performance of the thrust bearing. Chang and Jeng [5] researched the performance of aerostatic bearing by optimizing the bearing diameter, orifice diameter, orifice structure, gas supply pressure, and so on. Li et al. [6] proposed an optimization model by the maximum Mach number to improve dynamic stability. However, this is only one aspect of the optimization of aerostatic spindle.

Another aspect of research is that the final performance is determined by optimization methods. Shie and Shih [7] obtained a numerical solution of the pressure distribution between the surface of the aerostatic bearing and the worktable using the finite difference method. Li et al. [8,9] proposed a coupling optimization method to optimize the spindle structure by magnetic circuit calculation and magnetic field calculation. Wang and Chang [10] used Pareto ranking method and genetic algorithm to optimize the stiffness and air mass flow of porous air bearing. Besides, they used the hypercube-diving method to research the multiobjective optimization of porous air bearing [11]. These optimization methods have different objective function for the different structure of gas cavity and the aerostatic bearing with orifice has a high load capacity. The performance of gas film in microscale is different from the macroscale, and the 
corresponding optimization in microscale of structure of gas film is seldom.

In this paper, an optimization method is proposed to optimize the factors affecting the performance of the aerostatic spindle. These factors include gas film thickness, diameter of orifice, and the diameter of stomatic distribution circle. The relationship between load capacity, stiffness, and the main influence factors determined by correlation analysis is deduced. The results are verified from static and dynamic characteristics. And the corresponding simulation and experiment of the bearing capacity and stiffness of the aerostatic spindle are carried out to achieve enhanced performance in widely application.

\section{Pressure Distribution of Aerostatic Spindle}

2.1. Structure of Aerostatic Spindle. The circular flat pad aerostatic bearing with eight orifice-type restrictor is shown in Figure 1. According to the structure characteristics and actual working situation of the aerostatic bearing, the main design parameters contain bearing clearance $h$, orifice diameter $d_{3}$, the diameter of orifice distribution circle $d_{2}$, the rotate speed of the spindle, and so on. The bearing clearance $h$, orifice diameter $d_{3}$, and the diameter of orifice distribution circle $d_{2}$ are considered as design variables because they have more influence on the aerostatic bearing performance, and the ranges are $5-20 \mu \mathrm{m}$ for $h, 2 \mathrm{~mm}-5 \mathrm{~mm}$ for $d_{3}$, and $75-85 \mathrm{~mm}$ for $d_{2}$, respectively. The air supply pressure $p_{\mathrm{s}}$ is given between $0.3 \mathrm{MPa}$ and $0.6 \mathrm{MPa}$.

2.2. Flow Field Analysis in Microscale. Microfactors will lead to decrease of the gas film density and increase of the gas compressibility. Therefore, the traditional Reynolds equation is no longer applicable to describe the microscale flow. The flow factor $Q$ embodied microfactor whose equation is $Q=C_{1}+C_{2} / P H$ is introduced into the Reynolds equation, where $C_{1}$ and $C_{2}$ can be obtained from the literature [12]. So, the Reynolds equation after introducing the flow factor $Q$ is as follows:

$\frac{\partial}{\partial x}\left(p h^{3} Q \frac{\partial p}{\partial x}\right)+\frac{\partial}{\partial z}\left(p h^{3} Q \frac{\partial p}{\partial z}\right)=6 \mu U \frac{\partial(p h)}{\partial x}+12 \mu \frac{\partial(p h)}{\partial t}$,

where $p$ is the film pressure, $h$ is the bearing film thickness, $\mu$ is the dynamic viscosity of the lubricating gas, $U$ is the circumferential speed of the bearing surface, and $U=\omega R_{0}$. $\omega$ is the angular velocity of spindle rotation, $R_{0}$ is the bearing radius, and $t$ is the time. Equation (1) is converted into the polar coordinate for the convenience of calculation.

$$
\frac{\partial}{\partial r}\left(p h^{3} Q \frac{\partial p}{\partial r}\right)+\frac{\partial}{r^{2} \partial \theta}\left(p h^{3} Q \frac{\partial p}{\partial \theta}\right)=6 \mu U \frac{\partial(p h)}{\partial r}+12 \mu \frac{\partial(p h)}{\partial t} \text {. }
$$

Equation (2) is dimensionless for the convenience of calculation. The characteristic pressure of the film is $p_{0}$ and the characteristic thickness of film is bearing radius clearance $h_{0}$. Let $p=p_{0} P, p_{0}=\left(\left(6 \mu R_{0}^{2}\right) / h_{0}^{2}\right), \quad r=R_{0} R$, and $h=h_{0} H$. The characteristic length of the distribution circle radius of the orifice is $R$. $P$ is nondimensional pressure, and $H$ is nondimensional bearing film thickness. The time term is ignored in solving the static characteristic of bearing. So, the dimensionless form of Reynolds equation with flow factor $Q$ is as follows:

$$
\frac{\partial}{\partial R}\left(P H^{3} Q \frac{\partial P}{\partial R}\right)+\frac{\partial}{R^{2} \partial \theta}\left(P H^{3} Q \frac{\partial P}{\partial \theta}\right)=\frac{\omega \partial(P H)}{\partial R} .
$$

Two key steps for solving Reynolds equation by finite difference method are (1) dividing the solution domain into nodal grids and (2) transforming partial differential Reynolds equation into difference equation. Finally, the pressure distribution of $p_{a}$ can be obtained by substituting boundary conditions, as shown in Figure 2.

Then, use the disturbance method to establish unsteady Reynolds equation. The basic idea is to assume that when the input disturbance is a sinusoidal or cosine function, the output is the superposition of sinusoidal and cosine functions when the object of study is a first-order linear system. So, the integral of the pressure disturbance term in phase with the input disturbance can be defined as dynamic stiffness, and the integral of the pressure disturbance term with phase lag can be defined as dynamic damping coefficient.

$$
\left\{\begin{array}{l}
h=h_{0}+h_{0} \sin \omega_{1} t \\
p=p_{a}+\left(p_{b} \sin \omega_{1} t+p_{c} \cos \omega_{1} t\right)
\end{array}\right.
$$

where $p_{a}$ is the film pressure in the steady state, $h_{0}$ is steadystate film thickness, $\omega_{1}$ is the perturbation frequency, $p_{b}$ is the pressure perturbation term in phase with the input perturbation, and $p_{c}$ is the pressure perturbation term with phase lag. Substituting equation (4) in equation (1), let $p_{A}=p_{a} \cdot p_{b}, p_{B}=p_{a} \cdot p_{c}$, the disturbance quantities in quadratic and above are removed. The perturbed Reynolds equation is as follows:

$$
\left\{\begin{array}{l}
\frac{\partial^{2} p_{A}}{\partial r^{2}}+\frac{1}{r} \frac{\partial p_{A}}{\partial r}+\frac{1}{r^{2}} \frac{\partial^{2} p_{A}}{\partial \theta^{2}}=-\frac{12 \mu \omega_{1} P_{B}}{p_{a} h_{0}^{2}} \\
\frac{\partial^{2} p_{B}}{\partial r^{2}}+\frac{1}{r} \frac{\partial p_{B}}{\partial r}+\frac{1}{r^{2}} \frac{\partial^{2} p_{B}}{\partial \theta^{2}}=-\frac{12 \mu \omega_{1}\left(p_{a}^{2}+p_{A}\right)}{p_{a} h_{0}^{2}} .
\end{array}\right.
$$

The dynamic performance is solved by finite difference method. First, the perturbation Reynolds equation is discretized and then meshed. Finally, the boundary conditions of steady state and perturbation are introduced into the equation to solve the problem. By substituting the steady pressure $p_{a}$ and the steady film thickness $h_{0}$ obtained from the steady-state solution into the perturbation equation, the dynamic pressure and the dynamic film gap can be obtained. $p_{b}$ and $p_{c}$ can be obtained by solving equation (5).

2.3. Performance Analysis of Aerostatic Spindle. According to the pressure distributions, the load capacity $W$ is calculated by integrating the pressure along the lower surface of bearing. 


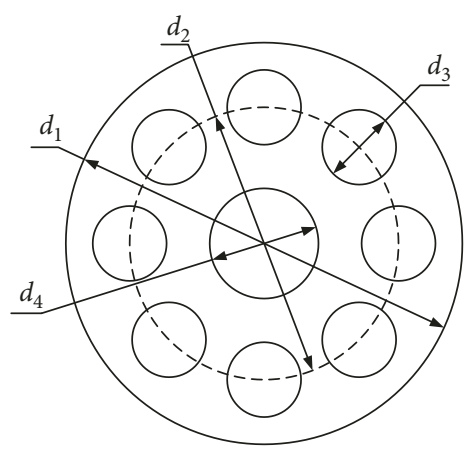

(a)

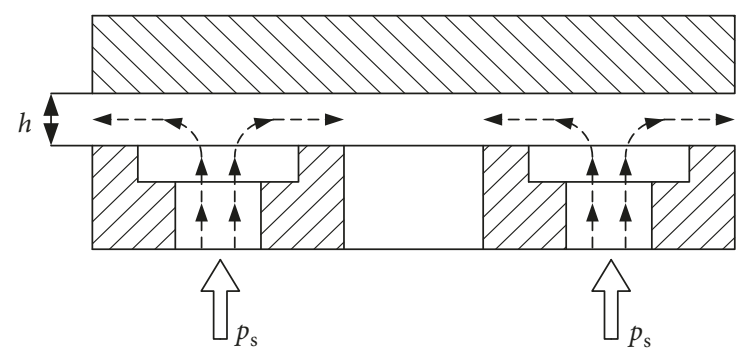

(b)

Figure 1: Structure of bearing.

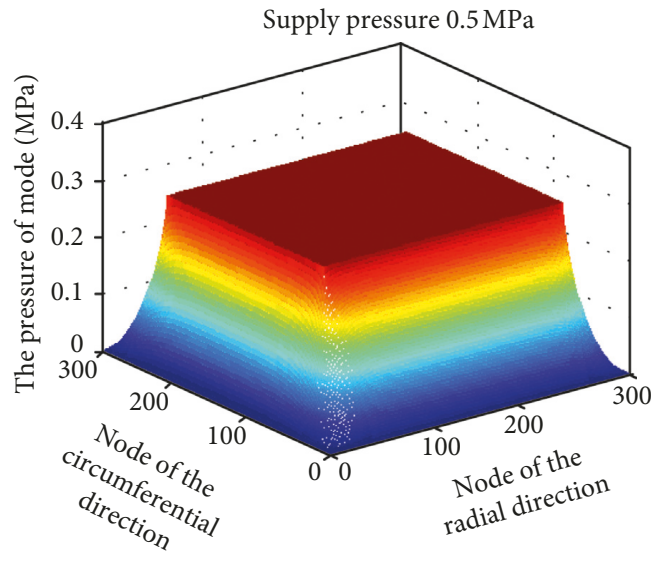

(a)

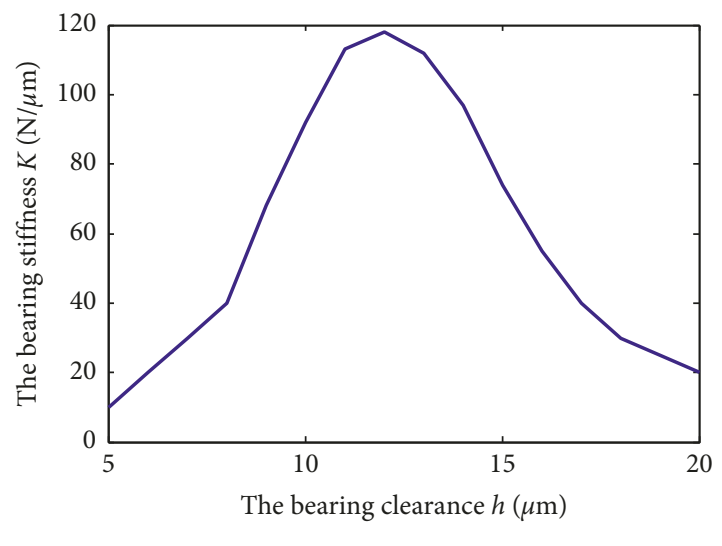

(b)

FIgURE 2: Performance of aerostatic spindle in axial direction. (a) Gas pressure distribution. (b) Relation between bearing stiffness and the gas film clearance.

$$
W=8 \int_{0}^{\pi / 4} \int_{d_{1 / 2}}^{d_{4 / 2}} \operatorname{pr} d r d \theta
$$

At the same time, the stiffness of bearing can be obtained by calculating the increment ratio of the bearing capacity and bearing clearance.

$$
K=\frac{d W}{d h}
$$

The relationship between stiffness and bearing clearance is shown in Figure 2(b). The bearing capacity and stiffness of the aerostatic spindle are affected by many factors, including structural and working parameters. Next, we will evaluate the effect of the main factors on the spindle performance.

\section{Degree Analysis of the Influence Factors of the Aerostatic Bearing}

3.1. Influence Factors on the Performance of Aerostatic Spindle. Theoretical calculations show that the factors affecting performance of aerostatic spindle include the bearing clearance $h$, orifice diameter $d_{3}$ and the diameter of orifice distribution circle $d_{2}$, the depth of the air cavity $d$ and the pressure supply $p_{s}$. However, the influence extent of these factors is different. For example, some factors improve the performance of the aerostatic bearing, and others reduce the performance. Therefore, the first step is to assess the influence extent of these factors.

3.2. Analysis of the Impact Extent of Influence Factors. In order to analyze the influence of the single factor more intuitively on the final performance of aerostatic bearing, the cross-correlation analysis method is proposed, which uses similarity of two series as a function of the lag of one relative to the other to evaluate correlation extent of the single parameter on the performance of aerostatic spindle. This is also known as a sliding dot product or sliding inner-product. To characterize the correlation between two random variables $x$ and $y_{1,2}$, which produce yield data sets $x=\left\{x_{1}, \ldots, x_{n}\right\}$ and $y_{1,2}=\left\{y_{1}, \ldots, y_{n}\right\}$, the correlation coefficient is usually used to express the degree of linear correlation between the two variables [13]. 


$$
R_{x y k}=\frac{\sum_{i=1}^{n}\left(x_{i}-\bar{x}\right)\left(y_{k i}-\overline{y_{k}}\right)}{\sqrt{\sum_{i=1}^{n}\left(x_{i}-\bar{x}\right)^{2}} \sqrt{\sum_{i=1}^{n}\left(y_{k i}-\overline{y_{k}}\right)^{2}}} \quad k=1,2,
$$

where $x$ is the influence factors including the bearing clearance $h$, orifice diameter $d_{3}$, the diameter of stomatic distribution circle $d_{2}$, the depth of the air cavity $d$, and the pressure supply $p_{\mathrm{s}}$. The ranges are $5 \mu \mathrm{m}-20 \mu \mathrm{m}$ for $h, 2 \mathrm{~mm}-5 \mathrm{~mm}$ for $d_{3}, 75 \mathrm{~mm}-85 \mathrm{~mm}$ for $d_{2}$, $0.75 \mathrm{~mm}-1.0 \mathrm{~mm}$ for $d$, and $0.3 \mathrm{MPa}-0.6 \mathrm{MPa}$ for $p_{\mathrm{s}} . i$ is the signal number of correlation analysis and $y_{1}$ and $y_{2}$ are the load capacity and stiffness of aerostatic spindle, respectively.

After analysis the above, the influence extent of single factor on the stiffness of the aerostatic bearing is obtained, as shown in Figure 3. From this, we can get that the correlation coefficients of the influence factors $h, d_{3}$, and $d_{2}$ are all more than 0.7 ; however, the correlation values of influence factor $d$ and $p_{\mathrm{s}}$ are both less than 0.6, which indicates the main influence factors are the bearing clearance $h$, orifice diameter $d_{3}$, and the diameter of stomatic distribution circle $d_{2}$. Therefore, next, we will optimize the three parameters.

\section{Numerical Model}

4.1. Relationship between Performance and Influence Factors. In order to find the relationship between the performance of aerostatic spindle and the influence factors. An array $X$ with these design variables which include the bearing clearance $h$, orifice diameter $d_{3}$, and the diameter of stomatic distribution circle $d_{2}$ is determined, $X=$ $\left[x_{1}, x_{2}, x_{3}\right]=\left[h, d_{3}, d_{2}\right]$. The industrial applications require high bearing capacity and stiffness, but their values vary with design variables. Therefore, the multiobjective optimization containing the bearing capacity and stiffness is proposed, and the multiobjective optimization functions $F(x)$ including the maximum bearing capacity function $f_{1}(x)$ and the maximum stiffness function $f_{2}(x)$ are established.

$$
F(x)=N_{1} f_{1}(x)+N_{2} f_{2}(x)
$$

where $N_{1}$ and $N_{2}$ represent weighting factors which reflect the specific gravity of bearing capacity and stiffness in the total objective function, respectively. And, they are calculated by the equation:

$$
N_{i}=\frac{1}{f_{i}\left(X^{*}\right)}
$$

where $f_{i}\left(X^{*}\right)$ represents the optimal solution of single constraint problem for the $i$-th subobjective as the objective function.

4.2. Function Determination of the Performance of Aerostatic Spindle. According to the assumption of uniform radial flow of gas, the motion equation in cylindrical coordinates can be simplified as follows:

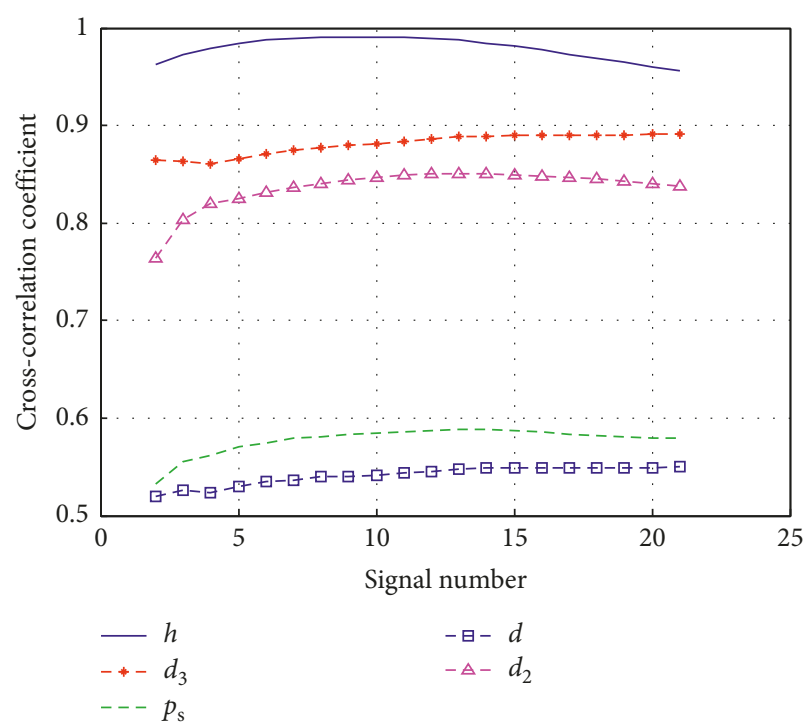

FIgURE 3: Correlation coefficient of the stiffness and influence factors.

$$
\begin{aligned}
& \frac{\partial p}{\partial r}=\mu \frac{\partial^{2} v_{r}}{\partial z^{2}} \\
& \frac{\partial p}{\partial \theta}=0 \\
& \frac{\partial p}{\partial z}=0
\end{aligned}
$$

By integrating equation (11) with $z$ twice and combining the equivalent mass flow equation and the gas state equation, we can get the expression of pressure $p$ as follows:

$$
\begin{array}{ll}
p_{1}^{2}=\left\{p_{a}^{2}-\left(p_{a}^{2}-p_{\mathrm{s}}^{2}\right) \frac{\ln \left(r / d_{4}\right)}{\ln \left(d_{2} / d_{4}\right)}\right\}^{1 / 2}, & d_{4}<r<d_{2}, \\
p_{2}^{2}=\left\{p_{0}^{2}-\left(p_{0}^{2}-p_{a}^{2}\right) \frac{\ln \left(r / d_{2}\right)}{\ln \left(d_{1} / d_{2}\right)}\right\}^{1 / 2}, & d_{2}<r<d_{1} .
\end{array}
$$

The expression of bearing capacity can be obtained by substituting equations (12) and (13) in equation (6):

$$
\begin{aligned}
W= & \left(p_{a}-p_{\mathrm{s}}\right) \frac{\pi\left(d_{2}^{2}-d_{4}^{2}\right)}{2 \ln \left(d_{2} / d_{4}\right)}+\left(p_{0}-p_{a}\right) \frac{\pi\left(d_{1}^{2}-d_{2}^{2}\right)}{2 \ln \left(d_{1} / d_{2}\right)} \\
& -\pi\left(d_{1}^{2}-d_{4}^{2}\right) p_{0}+\pi d_{3}^{2} p_{a} .
\end{aligned}
$$

Then, we can get $f_{1}(x)$.

$$
\begin{aligned}
f_{1}(x)= & \left(p_{a}-p_{s}\right) \frac{\pi\left(x_{3}^{2}-d_{4}^{2}\right)}{2 \ln \left(x_{3} / d_{4}\right)}+\left(p_{0}-p_{a}\right) \frac{\pi\left(d_{1}^{2}-x_{3}^{2}\right)}{2 \ln \left(d_{1} / x_{3}\right)} \\
& -\pi\left(d_{1}^{2}-d_{4}^{2}\right) p_{0}+\pi x_{2}^{2} p_{a} .
\end{aligned}
$$

And then, we define the gauge pressure ratio by using Powell's method. 


$$
k_{g}=\frac{p_{a}-p_{0}}{p_{\mathrm{s}}-p_{0}} .
$$

So, we write the bearing capacity as follows:

$$
\begin{aligned}
W= & k_{g}\left(p_{a}-p_{s}\right) \frac{\pi\left(d_{2}^{2}-d_{4}^{2}\right)}{2 \ln \left(d_{2} / d_{4}\right)}+\left(p_{0}-p_{a}\right) \frac{\pi\left(d_{1}^{2}-d_{2}^{2}\right)}{2 \ln \left(d_{1} / d_{2}\right)} \\
& -\pi\left(d_{1}^{2}-d_{4}^{2}\right) p_{0}+\pi d_{3}^{2} p_{a} .
\end{aligned}
$$

Then, we introduce the slit factor $G$.

$$
\begin{aligned}
G= & \frac{p_{a} / p_{\mathrm{s}}}{\left(1-p_{a} / p_{\mathrm{s}}\right)^{1 / 2}\left(1+p_{a} / p_{\mathrm{s}}\right)} \cdot \frac{24 \mu(2 R T)^{1 / 2}}{p_{a}} \\
& \cdot \frac{C_{\mathrm{D}} d_{3}^{2} \ln \left(d_{1} / d_{2}\right)}{8 h^{3}}, \\
k_{g}= & \frac{2}{1+\left(1+4 / G^{2}\right)^{1 / 2}} .
\end{aligned}
$$

According to the equation (7), we can get the objective function $f_{2}(x)$ of the stiffness as follows:

$$
f_{2}(x)=\frac{-0.98}{x_{1}}\left(p_{s}-p_{a}\right) \frac{\pi}{2} x_{2}\left[\frac{x_{3}^{2}-d_{4}^{2}}{\ln \left(x_{3} / d_{4}\right)}\right],
$$

where $x_{1}$ represents the gas film thickness $h, x_{2}$ is the orifice diameter $d_{3}$, and $x_{3}$ is the diameter of distribution circle $d_{2}$. $d_{1}$ represents the external diameter of bearing, $d_{4}$ represents the inner diameter of bearing, and $p_{\mathrm{s}}$ represents the air supply pressure. $p_{0}$ represents the atmospheric pressure and the discharge coefficient $C_{\mathrm{D}}=0.8$.

\section{Optimization Process}

5.1. Optimization of Single Objective Function. Using equation (15) as the fitness function to write the $M$ file program for optimizing the bearing capacity function. The constrains are also transformed to the program of $\mathrm{M}$ file, that is, the ranges of $h, d_{3}$ and $d_{2}$ are 5-20 $\mu \mathrm{m}, 2 \mathrm{~mm}-5 \mathrm{~mm}$ and $75-$ $85 \mathrm{~mm}$ respectively. The $\mathrm{M}$ files reflecting the bearing capacity and constrains are imported to the genetic algorithm toolbox of MATLAB software. The process of solution is shown in Figure 4. The result of optimization is shown in Figure 5. When the design variable $X=\left[\begin{array}{lll}8.301 & 3.213 & 82.531\end{array}\right]$, that is, bearing clearance is $8.301 \mu \mathrm{m}$, orifice diameter is $3.213 \mathrm{~mm}$, and the diameter of stomatic distribution circle is $82.531 \mathrm{~mm}$, the optimal result of single objective function $f_{1}(x)$ is obtained. The optimal value of the bearing capacity is $\max f_{1}(x)=1254.2$.

Use equation (20) as another fitness function to write the $\mathrm{M}$ file program for optimizing the stiffness function. The constrains are also transformed to the program of $\mathrm{M}$ files. The $\mathrm{M}$ files reflecting the stiffness and constrains are imported to the genetic algorithm toolbox. The process of solution is shown in Figure 6. The result of optimization is shown in Figure 7 . When the design variable $X=[12.342$ $2.91282 .125]$, that is, bearing clearance, stomatal diameter,

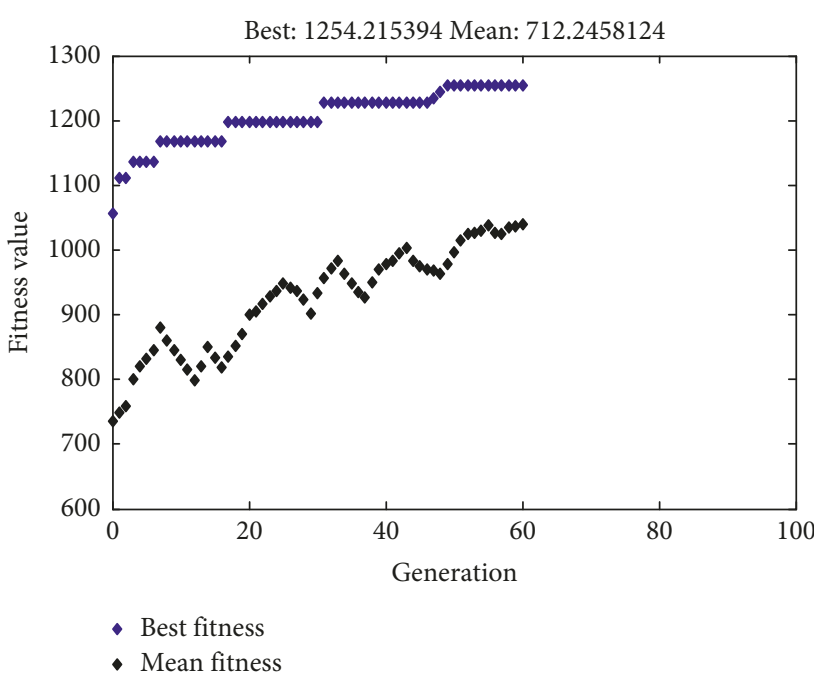

Figure 4: Import of goal function and constrains.

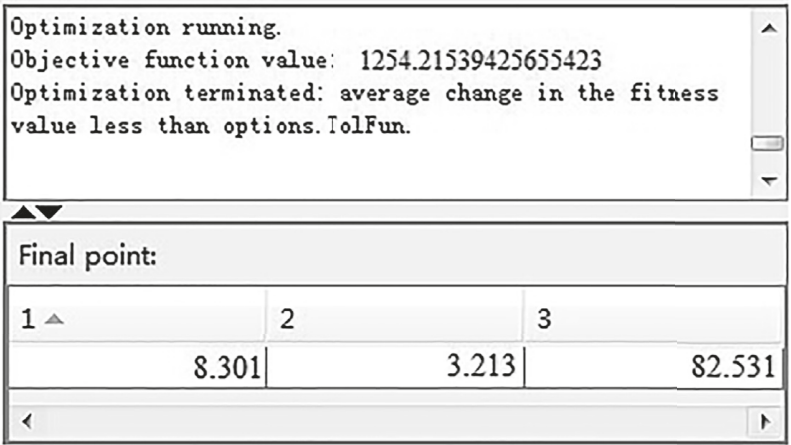

FIGURE 5: Optimization results of capacity.

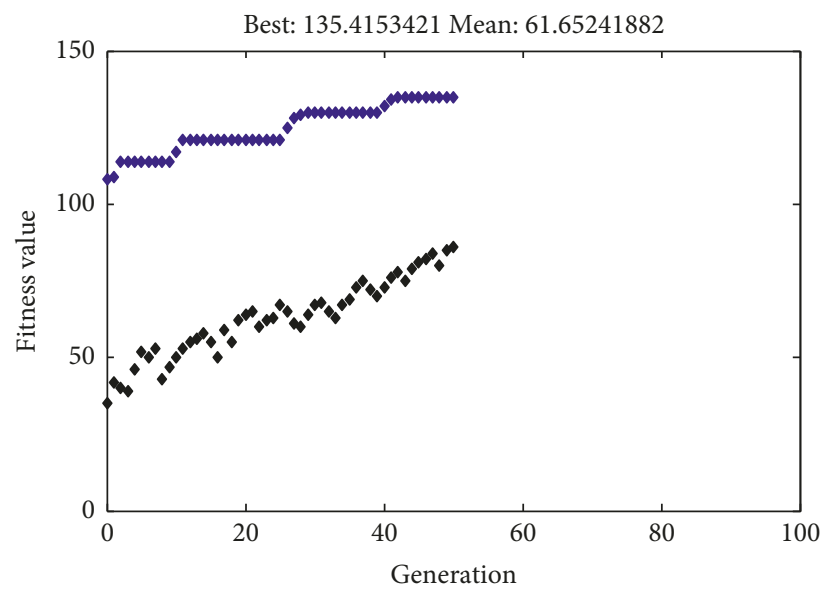

- Best fitness

- Mean fitness

Figure 6: Import of goal function and constrains.

and the diameter of stomatic distribution circle are $12.342 \mu \mathrm{m}, 2.912 \mathrm{~mm}$, and $82.125 \mathrm{~mm}$ respectively, the optimal result of single objective function $f_{2}(x)$ is 


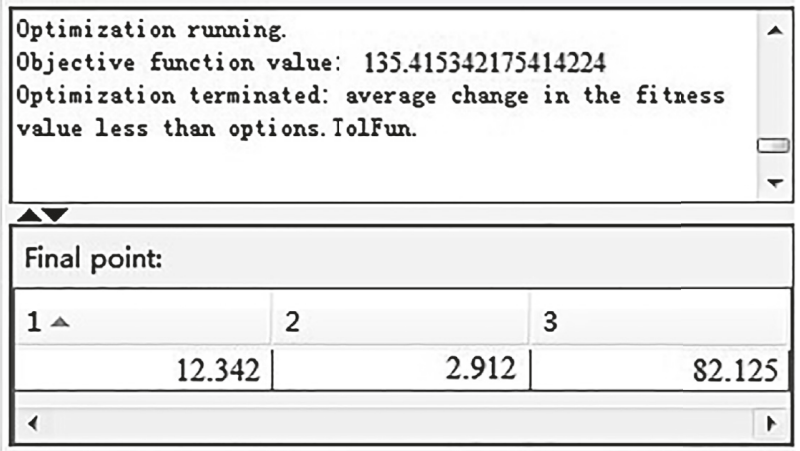

Figure 7: Optimization results of stiffness.

obtained. The optimal value of the stiffness is max $f_{2}(x)=135.4$.

The bearing capacity and stiffness of aerostatic spindle are optimized by using genetic algorithm toolbox in simulation system software that combines the optimization theory and genetic algorithm. First, the performance of aerostatic bearing is optimized by the single objective function of the capacity and the stiffness. The optimization results are shown in Table 1.

It can be seen from Table 1 that the contradiction is present in the optimal result of the stiffness and load capacity function, respectively. Thus multiobjective optimization function is used to optimize the performance of the aerostatic spindle in order to get ideal consistent results.

5.2. Optimization of Multiobjective Function. The optimal values of bearing capacity and stiffness calculated above are brought into equation (10) to obtain the weighting factors $N_{1}$ and $N_{2}$. Substituting formulas (15) and (19) in (9), the multiobjective function including bearing capacity, stiffness, and influence parameters of aerostatic bearing is determined.

$$
F(X)=0.00079 f_{1}(X)+0.00741 f_{2}(X) .
$$

The fitness function is shown in formula (12). The multiobjective function and constrains are imported to the genetic algorithm toolbox in the simulation. The optimization process of the solution is shown as Figure 8.

The result of optimization is shown in Figure 9. When the design variable $X=\left[\begin{array}{lll}10.521 & 3.501 & 82.214\end{array}\right]$, that is, bearing clearance is $10.521 \mu \mathrm{m}$, orifice diameter is $3.501 \mathrm{~mm}$, and the diameter of stomatic distribution circle is $82.214 \mathrm{~mm}$, the optimal result of multiobjective function $F(X)$ is max $F(X)=1.8682$.

\section{Discussion}

6.1. Comparison of Static Performance by Optimization Parameters. The comparison of optimization parameters obtained by multiobjective function $F(X)$ and original parameters are shown in Table 2. Using optimization parameters and original parameters, the bearing capacity and stiffness of the different bearing clearance are simulated. The bearing performance before and after optimization is shown
TABLE 1: Optimal results of single objective function.

\begin{tabular}{lccc}
\hline \multirow{2}{*}{ Optimization values } & \multicolumn{3}{c}{ Optimization parameters } \\
& $h(\mu \mathrm{m})$ & $d_{3}(\mathrm{~mm})$ & $d_{2}(\mathrm{~mm})$ \\
\hline$W=1254.2 \mathrm{~N}$ & 8.301 & 3.213 & 82.531 \\
$K=135.4 \mathrm{~N} / \mu \mathrm{m}$ & 12.342 & 2.912 & 82.125 \\
\hline
\end{tabular}

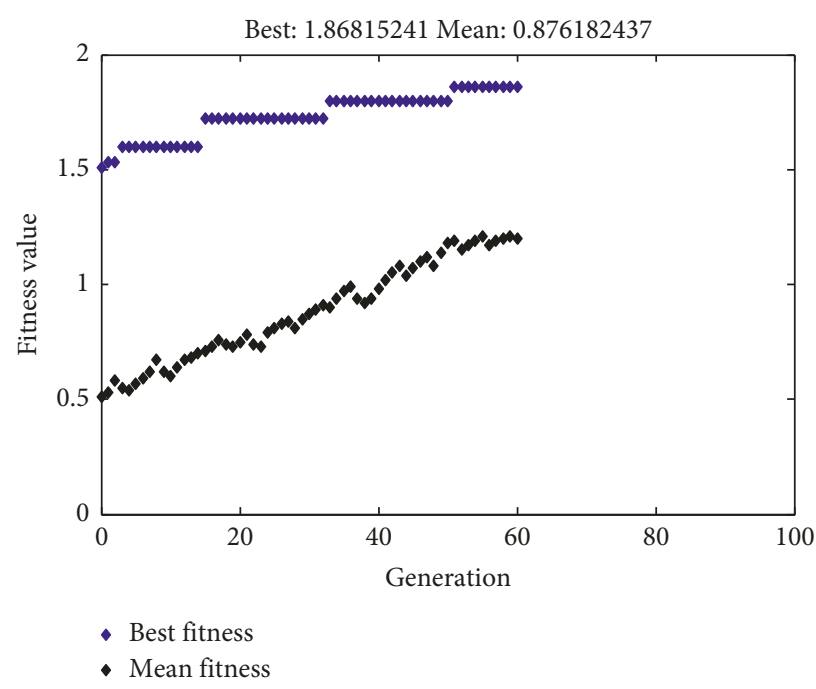

FIGURE 8: Optimization process.

\begin{tabular}{|c|c|c|c|}
\hline \multicolumn{3}{|c|}{$\begin{array}{l}\text { Optimization running. } \\
\text { Objective function value: } 1 . \$ 6 \$ 15241155324632 \\
\text { Optimization terminated: average change in the fitness } \\
\text { value less than options. TolFun. }\end{array}$} & \\
\hline \multicolumn{4}{|l|}{$\Delta \nabla$} \\
\hline \multicolumn{4}{|l|}{ Final point: } \\
\hline 14 & 2 & 3 & \\
\hline 10.521 & 3.501 & 82.2 & \\
\hline 1 & & & 1 \\
\hline
\end{tabular}

Figure 9: Optimization results of multiple objective.

TABLE 2: Comparison of optimization parameters and original parameters.

\begin{tabular}{lccccc}
\hline & $\begin{array}{c}h \\
(\mu \mathrm{m})\end{array}$ & $\begin{array}{c}d_{3} \\
(\mathrm{~m})\end{array}$ & $\begin{array}{c}d_{2} \\
(\mathrm{~mm})\end{array}$ & $\begin{array}{c}W \\
(\mathrm{~N})\end{array}$ & $K(\mathrm{~N} / \mu \mathrm{m})$ \\
\hline $\begin{array}{l}\text { Original parameters } \\
\begin{array}{l}\text { Optimization } \\
\text { parameters }\end{array}\end{array}$ & 12 & 3.5 & 82.5 & 1052 & 120 \\
\hline
\end{tabular}

in Figures 10 and 11. Compared with original parameters, the performance of bearing with optimized parameters is improved. The bearing capacity and the stiffness are increased $7.98 \%$ and $9.17 \%$, respectively.

The aerostatic spindle system and the static stiffness measurement system of aerostatic bearing are, respectively, established by the optimization parameters of the bearing, as 


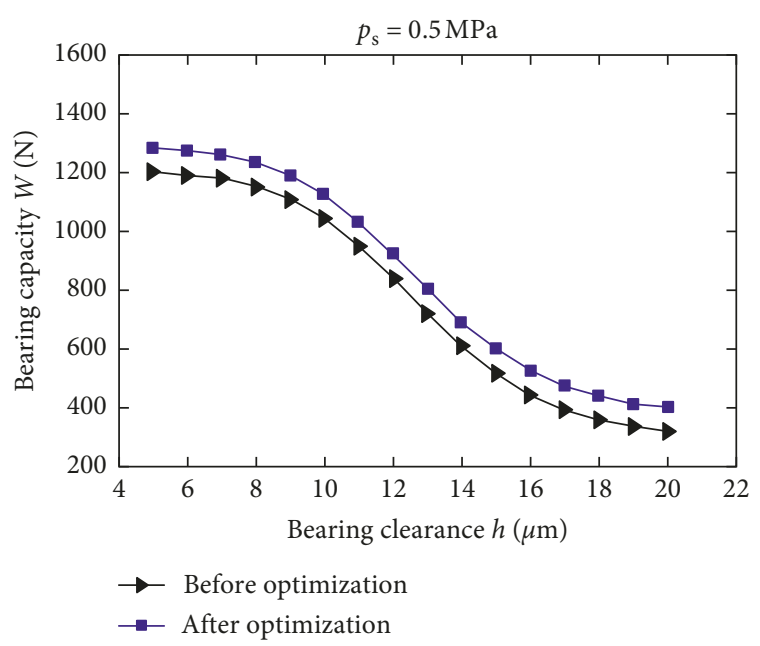

Figure 10: Comparison of bearing capacity.

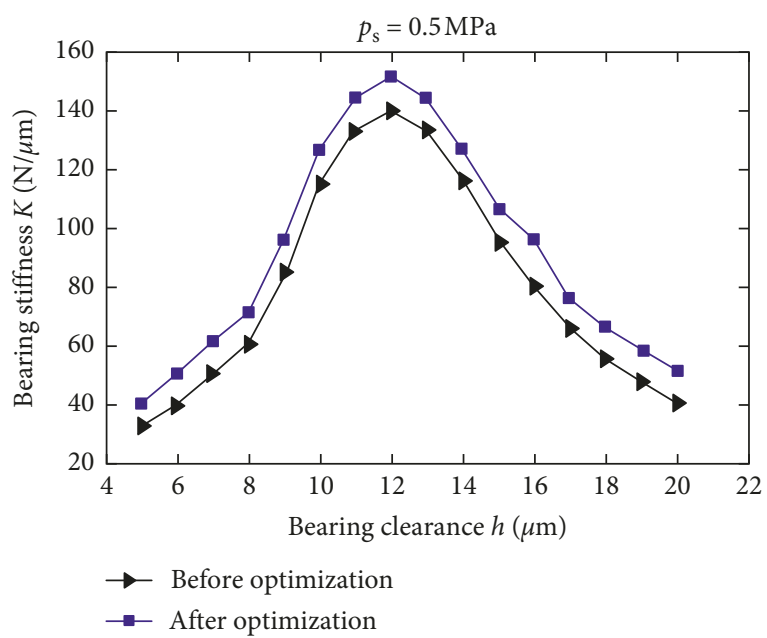

Figure 11: Comparison of bearing stiffness.

shown in Figure 12. An inductive micrometer is used to measure the variation of the displacement of the spindle table, and it is necessary to perform zero adjustment first. The specific operation is to determine the height of the induction microprobe by adjusting the height of the measuring gantry. The contact between the probe and the table is within $\pm 2 \mu \mathrm{m}$ to ensure the accuracy of the test.

Air compressor provides gas to the aerostatic spindle system, and the supply pressure is $0.5 \mathrm{MPa}$. When the mass block as load is applied to worktable of aerostatic spindle, the workbench will have vertical displacement change. The change is sensed by the probe of the inductive micrometer and transmitted to the inductive micrometer which shows the change in display. The weight of mass block is $50 \mathrm{~N}$, and the method of stepwise loading is used to perform multiple measurements and average. According to loaded load of the aerostatic bearing and the displacement change detected by the inductance micrometer, the static stiffness value is calculated by the stiffness calculation formula. The values of stiffness test are shown in Figure 13 which shows that the

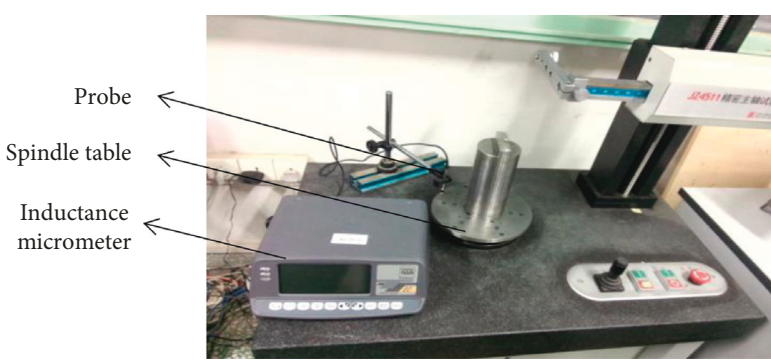

FIgURE 12: Measurement system of aerostatic bearing static stiffness.

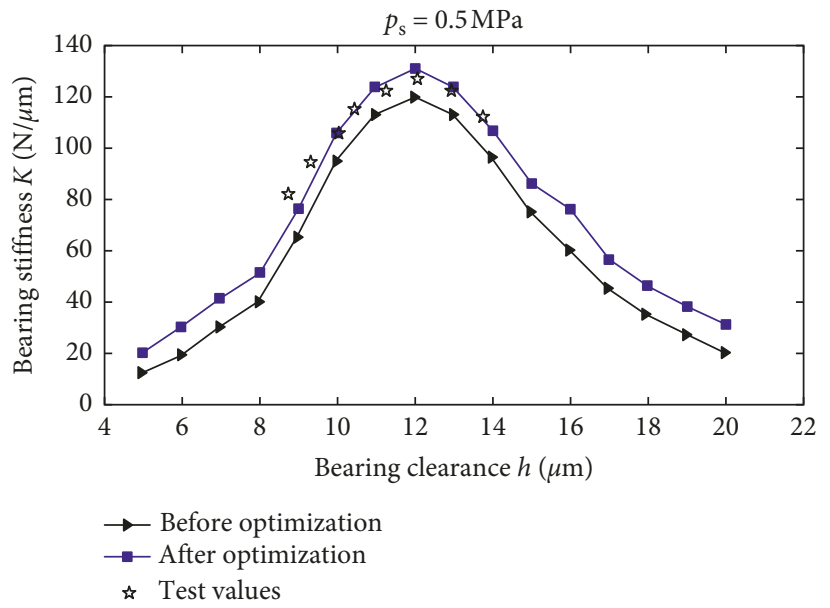

Figure 13: Comparison of bearing stiffness.

stiffness after optimization is more close to the values of stiffness test. So, the correctness and the feasibility of optimization method in this paper are verified by stiffness test.

6.2. Comparison of Dynamic Response by Optimization Parameters. By optimizing the parameters, the static performance of the aerostatic spindle is improved; however, the dynamic performance of the spindle can be affected. This is because the corresponding frequency varies with the change of bearing capacity and stiffness. Here, the COMBIN14 spring-damper unit is used in modal analysis of the spindle model, in which the thrust bearings can be seen as the longitudinal spring-damper. The node of each orifice in the gas cavity is only stretched or compressed and not affected by bending and torsion. The radial bearings can be seen as the longitudinal and torsional spring-damper, and the node of each orifice in the gas cavity has the rotary element with three degrees of freedom in addition to the tensioncompression in the normal direction. The model of aerostatic spindle is shown in Figure 14.

And the natural frequencies of the first six orders before and after optimization are calculated and shown in Table 3, in which we can get that the corresponding frequency has been increased $5 \%$ after optimization.

The dynamic characteristics of the aerostatic spindle are important in actual work, and the performance in axial direction is the key factor affecting the machining accuracy 


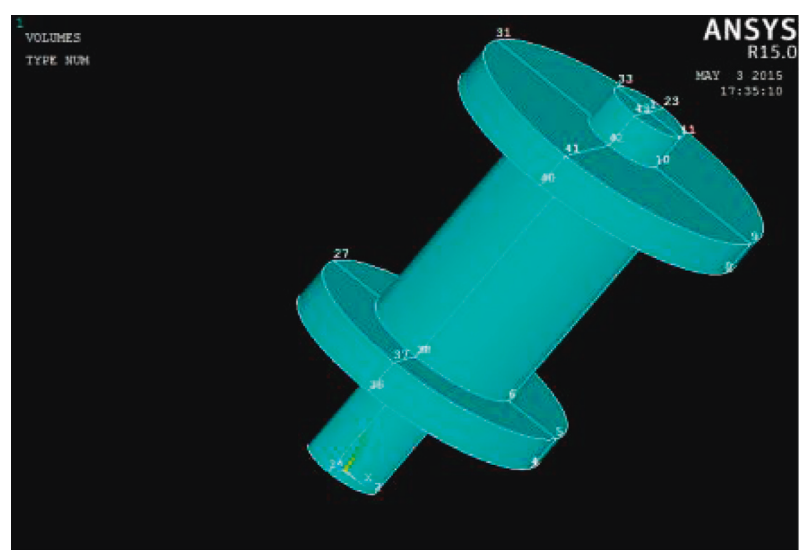

Figure 14: Model of aerostatic spindle.

TABLE 3: First 6-order frequency of spindle in two cases.

\begin{tabular}{lcccccc}
\hline Orders & 1 & 2 & 3 & 4 & 5 & 6 \\
\hline Original parameters $(\mathrm{Hz})$ & 494 & 496 & 1439 & 4857 & 4876 & 5591 \\
Optimization $(\mathrm{Hz})$ & 516 & 518 & 1503 & 5075 & 5094 & 5842 \\
\hline
\end{tabular}

of the machine tool. In order to better verify the optimization effect of aerostatic bearing, the axial dynamic response is analyzed. The motion equation which has natural frequency and damping coefficient is proposed.

$$
\begin{aligned}
\ddot{x}+2 \omega_{\mathrm{n}} \xi \dot{x}+\omega_{\mathrm{n}}^{2} x & =0, \\
\xi & =\frac{C}{2 m \omega_{\mathrm{n}}} .
\end{aligned}
$$

where $\omega_{\mathrm{n}}$ is the natural frequency, $m$ is the mass of the spindle, which is $3.7 \mathrm{~kg}$, and $C$ is the damping coefficient. The solution of above equation is as shown.

$$
x_{0}(t)=1-\frac{e^{-\xi \omega_{\mathrm{n}} t}}{\sqrt{1-\xi^{2}}} \sin \left(\omega_{\mathrm{d}} t+\arctan \frac{\sqrt{1-\xi^{2}}}{\xi}\right) .
$$

And the response curve is shown in Figure 15 which indicates that the response speed after optimization has been improved, but it still in the same order of magnitude with the values before optimization.

\section{Conclusions}

In this paper, the performance of aerostatic spindle has been improved by a novel optimization approach, and the results of before and after optimization are compared in static and dynamic aspect. In the optimization process, the main factors affecting the bearing capacity and stiffness of aerostatic bearings are determined by cross-correlation analysis, and the corresponding actual experimental platform of the aerostatic spindle system is established for analysis. The following major conclusions are obtained:

(1) A new optimization method for the performance of aerostatic spindle considering the main influence factors was proposed and proved experimentally.

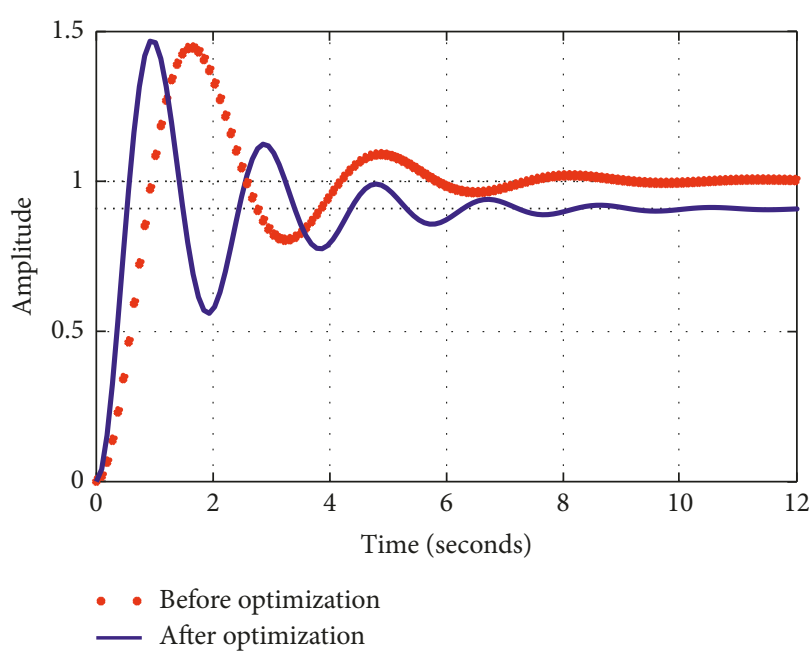

FIgURE 15: Dynamic response of aerostatic spindle.

Taking the load capacity and stiffness of the spindle as the optimize function in the multiobjective function. The relationship between the optimize function and the main influence factors is proposed. Then, the maximum values are obtained by genetic algorithm.

(2) The influence factors affecting the performance of the aerostatic spindle were evaluated, whose influence degree were obtained by cross-correlation analysis. And the correlation coefficients of more than 0.7 were determined, which are imported into the optimization process as the independent variables.

(3) The dynamic characteristics after optimization were analyzed, which show that the optimization method proposed in the paper provided effective parameters for the aerostatic spindle. The corresponding natural frequency and the response speed have been increased when the response amplitude is in the same order magnitude with the value before optimization. The optimization of aerostatic bearing is proceeded combining the theory with microscale characteristics. The performance of aerostatic bearing is improved.

\section{Data Availability}

The data used to support the findings of this study are included within the article.

\section{Conflicts of Interest}

The authors declare no conflicts of interest in preparing this article.

\section{Acknowledgments}

This research was funded by the National Natural Science Foundation of China (grant nos. 51875005 and 51475010), Beijing Nova Program (Z161100004916156), and Natural 
Science Foundation of Beijing Municipality (grant no. 3142005).

\section{References}

[1] S. J. Zhang, S. To, and H. T. Wang, "A theoretical and experimental investigation into five-DOF dynamic characteristics of an aerostatic bearing spindle in ultra-precision diamond turning," International Journal of Machine Tools and Manufacture, vol. 71, pp. 1-10, 2013.

[2] F. Al-Bender, "On the modelling of the dynamic characteristics of aerostatic bearing films: from stability analysis to active compensation," Precision Engineering, vol. 33, no. 2, pp. 117-126, 2009.

[3] S. S. Li, Y. H. Guo, Z. B. Zhu et al., "Static characteristics analysis and basic parameters optimization of aerostatic bearing for precision machine tool," Lubrication Engineering, vol. 37, no. 1, pp. 29-32, 2012.

[4] G. Aguirre, F. Al-Bender, and H. Van Brussel, "A multiphysics model for optimizing the design of active aerostatic thrust bearings," Precision Engineering, vol. 34, no. 3, pp. 507-515, 2010.

[5] S. H. Chang and Y. R. Jeng, "A modified particle swarm optimization algorithm for the design of a double-pad aerostatic bearing with a pocketed orifice-type restrictor," Journal of Tribology, vol. 136, no. 2, article 021701, 2013.

[6] Y. Li, Y. Yin, H. Yang, X. Liu, J. Mo, and H. Cui, "Modeling for optimization of circular flat pad aerostatic bearing with a single central orifice-type restrictor based on CFD simulation," Tribology International, vol. 109, pp. 206-216, 2017.

[7] J. S. Shie and M. C. Shih, "A study on optimization design and performance test of an aerostatic bearing," Advanced Materials Research, vol. 538-541, pp. 3182-3186, 2012.

[8] L. Y. Li, B. Q. Kou, J. W. Cao et al., "Optimization design of permanent magnet synchronous motor for the electrical spindle," Electrical Machines and Systems, vol. 2, pp. 205-207, 2008.

[9] L. Li, B. Kou, J. Cao et al., "Optimization design of permanent magnet synchronous motor for the electrical spindle," in Proceedings of the 11th International Conference on Electrical Machines and Systems, pp. 205-207, Institute of Electrical and Electronics Engineers, Wuhan, China, October 2008.

[10] N. Wang and Y.-Z. Chang, "Application of the genetic algorithm to the multi-objective optimization of air bearings," Tribology Letters, vol. 17, no. 2, pp. 119-128, 2004.

[11] N. Wang and K.-C. Cha, "Multi-objective optimization of air bearings using hypercube-dividing method," Tribology International, vol. 43, no. 9, pp. 1631-1638, 2010.

[12] B.-J. Shi and T.-Y. Yang, "Simplified model of Reynolds equation with linearized flow rate for ultra-thin gas film lubrication in hard disk drives," Microsystem Technologies, vol. 16, no. 10, pp. 1727-1734, 2010.

[13] W. H. Pres, S. A. Teukolsky, W. T. Vetterling, and B. P. Flannery, Numerical Recipes, Cambridge University Press, Cambridge, UK, 1992. 


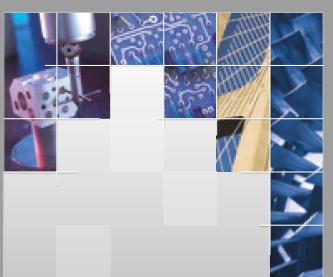

\section{Enfincering}
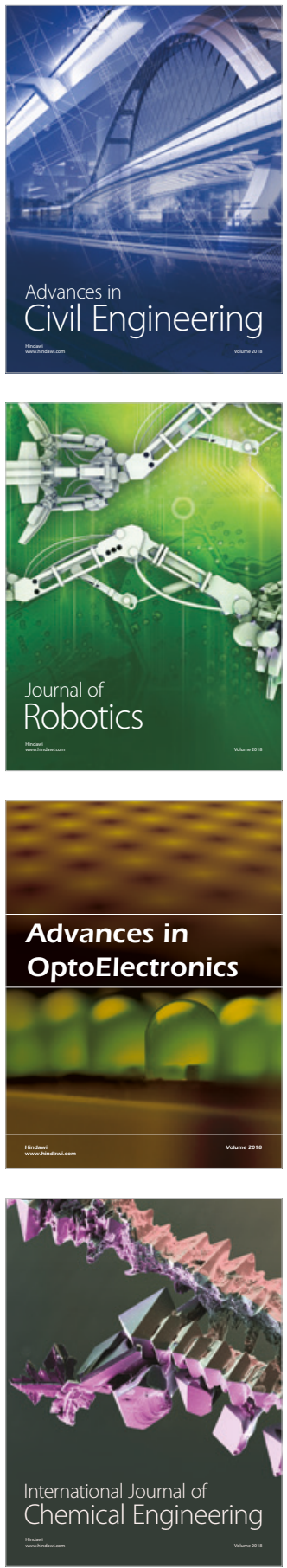

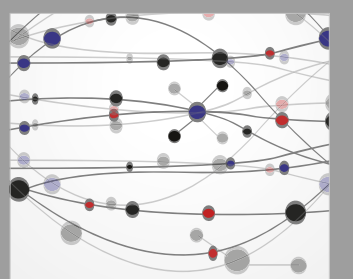

\section{Rotating \\ Machinery}

The Scientific World Journal

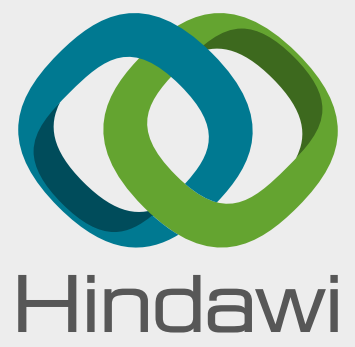

Submit your manuscripts at

www.hindawi.com
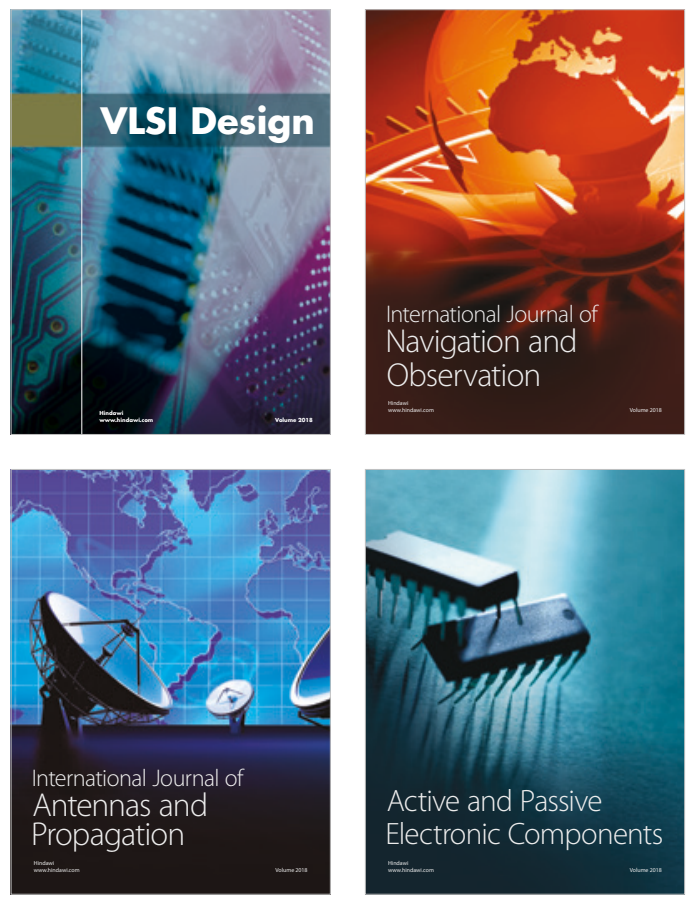
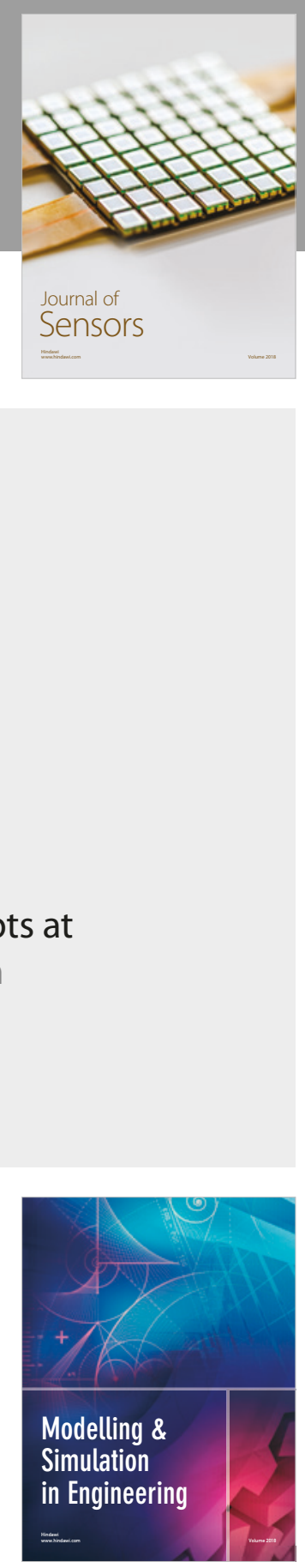

\section{Advances \\ Multimedia}
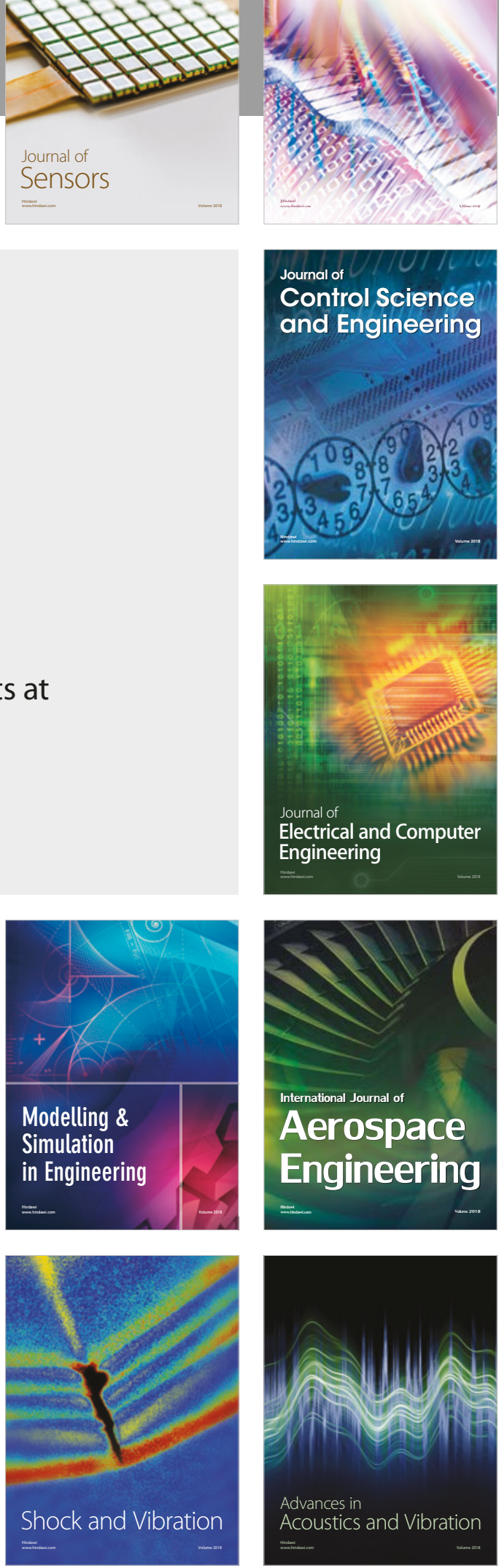